\title{
AN NUMERICAL METHOD BASED ITERATIVE PROCESS TO CHARACTERIZE MICROWAVE PLANAR CIRCUITS
}

\author{
Mohamed Tellache ${ }^{1)}$, Youcef Lamhene ${ }^{1)}$, Brahim Haraoubia ${ }^{1)}$, Henri Baudrand ${ }^{2)}$ \\ ${ }^{1)}$ Laboratory of Instrumentation (LINS), Faculty of Electronics and Computers, \\ USTHB, B.P 32, Bab-Ezzouar, 16111, Algiers, Algeria, http://www.usthb.dz \\ E-mail: tellachemoh@yahoo.fr, youcef_lamhene@yahoo.fr and haraoubiab@gmail.com, \\ ${ }^{2)}$ Laboratoire LAME, INP, ENSEEIHT \\ 2 Rue Charles Camichel, BP7122-31071, Toulouse Cedex 7, France, http://www. len7.enseeiht.fr \\ E-mail: baudranh@len7.enseeiht.fr
}

\begin{abstract}
In the present work, the modeling of microwaves planar circuits is proposed with an original method based on the Waves Concept Iterative Process (WCIP). It consists in the development of simulation software based on an iterative method. The iterative method is developed from the fast modal transform on a two-dimensional fast Fourier transform (FFT) algorithm. The method has been applied to the characterization and the modeling of patch antennas with notches in microstrip and coplanar technology and the quarter wavelength directive coupler. The obtained results are very powerful and successfully compared to others methods in term of time and reliability of convergence and particularly the accuracy of the results obtained in comparison with previous works.
\end{abstract}

Keywords: Iterative method, Modeling, Planar circuits, Coplanar circuits, Microwaves, Patch antennas, Quarter wavelength directive coupler.

\section{INTRODUCTION}

The planar circuits are characterized by there abilities to integrate important electronic elements. The global circuit modeling requires the development of simulation software that uses optimized numerical methods. In this paper we used an iterative method based on the wave concept. Among the most recent and the most efficient iterative methods the Wave Concept Iterative Process (WCIP) was developed at first as an instrument for the study of in-guide and planar circuits scattering problems [1].

The integral formulation of the transmission line modeling (TLM) method [2] is usually defined in the time domain but is expressed in the spectral domain in [3]. This spectral representation is the basis of a proposed procedure called the wave concept iterative procedure (WCIP). The same link that relates the TLM method to WCIP relates the electric or magnetic field integral equation to the finite difference time-domain techniques [4]. The WCIP is based on the determination of the recurrence relation between the incident and the reflected waves, with respect to the continuity conditions in the spatial domain.

The result is approached progressively by successive iterations until its convergence. This technique avoids the undesired phenomenon of unbounded operators; relations between currents and fields, obtained using unbounded impedance operators, are transposed to relations between waves, supplied by bounded scattering operators [5]. The method's convergence is therefore always guaranteed [6]. This method has been applied since one decade to many kinds of Radio Frequency Integrated Circuits (RFIC) and diffraction problems and the power of the WCIP was first verified in the analysis of simple planar circuits by Akatimagool et al. [7] and Wane et al. [8].

However, it is important to examine carefully the respective impact of the number of iterations and precision of pixellisation on the results of some sensible structures. Moreover, a very important gain in computation time is obtained by the use of the Two-Dimensional Fast Fourier transform (2DFFT) algorithm [9]. The method is applied to the characterization and modeling of patch antenna with notches in microstrip technology, coplanar antenna patch and finally a directive quarter wavelength coupler.

The results were compared with moments and finite difference time domain (FDTD) methods [10]. We notice that the iterative and moments method 
use planar sources (horizontal) and metallic walls, while the FDTD method uses vertical sources and absorbents walls. According to obtained results, a good efficiency and high spatial resolution is observed in spite of using weak resources. Good agreement between the simulation and measurement has been obtained.

\section{METHOD PRINCIPLES}

With the WCIP, the electromagnetic problem is solved in several steps [11]. First, the interfaces are characterized using space operators, and then the remaining space is represented using spectral operators. Finally, the electromagnetic sources involved in the model are added to the iterative process. Consider the diagram of figure 1 representing an example of a planar circuit printed on a dielectric substrate.

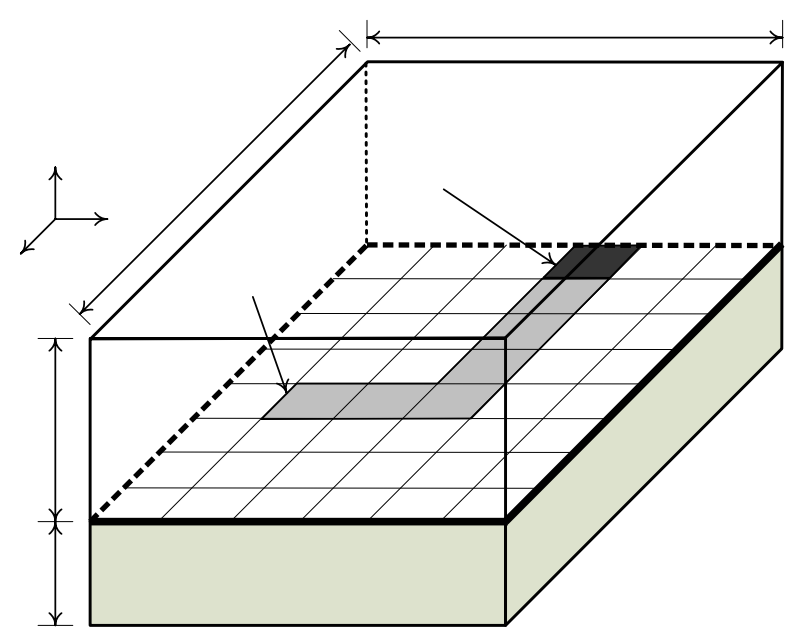

Fig.1 - The studied planar circuit

\subsection{WAVE CONCEPT}

$\Omega_{\mathrm{i}}$ ( $i$ stands for the medium 1 or 2 ), describes the boundaries interfaces on one side of the interface oriented with the normal vector $n_{i}$ toward the $i$ region. The current density $\vec{J}_{i}$ is defined by,

$$
\vec{J}_{i}=\vec{H}_{i} \times \vec{n}_{i}
$$

where $\vec{H}_{i}$ is the tangent magnetic field to the discontinuity of the interface corresponding to the field electric $\vec{E}_{i}$. The equation of wave [1] is defined by,

$$
\left\{\begin{array}{l}
\vec{A}_{i}=\frac{1}{2 \sqrt{Z_{0 i}}}\left(\vec{E}_{i}+Z_{0 i} \vec{J}_{i}\right) \\
\vec{B}_{i}=\frac{1}{2 \sqrt{Z_{0 i}}}\left(\vec{E}_{i}-Z_{0 i} \vec{J}_{i}\right)
\end{array}\right.
$$

where $\vec{A}_{i}$ the reflected waves, $\vec{B}_{i}$ the incident waves at the interface of discontinuity and $Z_{\mathrm{oi}}$ the impedance of wave in the medium 1 .

The equations of the electric field $\vec{E}_{i}$ and current density $\vec{J}_{i}$ are derived from the following equations,

$$
\left\{\begin{array}{l}
\vec{E}_{i}=\sqrt{Z_{o i}}\left(\vec{A}_{i}+\vec{B}_{i}\right) \\
\vec{J}_{i}=\frac{1}{\sqrt{Z_{o i}}}\left(\vec{A}_{i}-\vec{B}_{i}\right)
\end{array}\right.
$$

\subsection{THE ITERATIVE PROCESS}

The figure 2 shows the principle of the iterative process. It is based on the repetition of the equations process until problem resolution.

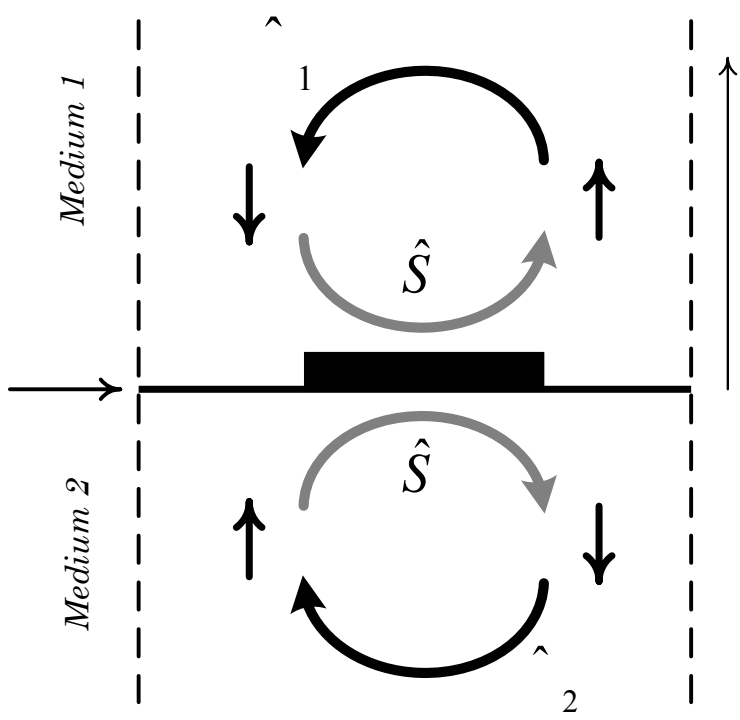

Fig. 2 - Iterative process scheme

This method is governed by the following relations [9],

$$
\left\{\begin{array}{l}
\vec{A}=€ \times \vec{B} \\
\vec{B}=£ \times \vec{A}+\vec{B}_{o}
\end{array}\right.
$$

where $€$ operator of reflection links the reflected waves to the incident waves in the spectral domain, $£$ operator of diffraction links the reflected waves to the incident waves in the spatial domain at the dielectric or metallic interface and $\vec{B}_{o}$ is the excitation wave source.

\section{II.3 THE FOURIER TRANSFORM}

Equations 5 and 6 give fundamental principle of the fast Fourier transform in direct and inverse 
domain.

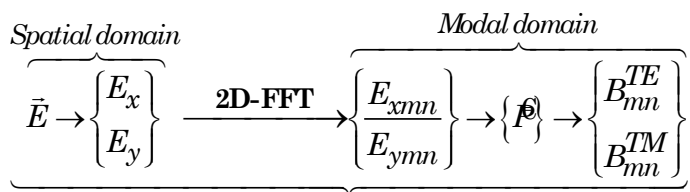

FMT

$$
\underbrace{\text { Spatial domain }}_{\vec{E} \leftarrow\left\{\begin{array}{c}
E_{x} \\
E_{y}
\end{array}\right\}} \stackrel{\text { 2D-FFT }^{-1}}{\overbrace{\left\{\begin{array}{l}
E_{x m n} \\
E_{y m n}
\end{array}\right\} \leftarrow\{f\}}^{\text {Modal domain }} \leftarrow\left\{\begin{array}{c}
B_{m n}^{T E} \\
B_{m n}^{T M}
\end{array}\right\}}
$$$$
\text { FMT }^{-1}
$$

In the spatial domain, the components of the electric field vector are defined analytically into two distinct bases [12], either according to the amplitudes of the fields in the Cartesian basis,

$$
\begin{aligned}
& \left|E_{x}(x, y)\right\rangle=\sum_{m, n} E_{x m n}\left|e^{-j \frac{2 \pi n}{a} x} e^{-j \frac{2 \pi m}{b} y}\right\rangle \\
& \left|E_{y}(x, y)\right\rangle=\sum_{m, n} E_{y m n}\left|e^{-j \frac{2 \pi n}{a} x} e^{-j \frac{2 \pi m}{b} y}\right\rangle
\end{aligned}
$$

According to the TE and TM amplitudes modes on the modal basis.

$$
\begin{aligned}
& \left|E_{x}(x, y)\right\rangle=\sum_{m, n} B_{m n}^{T E}\left|f_{x m n}^{T E}\right\rangle+\sum_{m, n} B_{m n}^{T M}\left|f_{x m n}^{T M}\right\rangle \\
& \left|E_{y}(x, y)\right\rangle=\sum_{m, n} B_{m n}^{T E}\left|f_{y m n}^{T E}\right\rangle+\sum_{m, n} B_{m n}^{T M}\left|f_{y m n}^{T M}\right\rangle
\end{aligned}
$$

The combination of equations (4) and (5) with other equations leads to the matrix relations, defining the transition operator from the spatial domain to the modal domain as follows:

$$
\begin{gathered}
{\left[\begin{array}{c}
E_{x m n} \\
E_{y m n}
\end{array}\right]=P^{-1}\left[\begin{array}{c}
B_{m n}^{T E} \\
B_{m n}^{T M}
\end{array}\right]} \\
\mathcal{E}^{-1}=\left[\begin{array}{cc}
K_{y m n} & K_{x m n} \\
-K_{x m n} & K_{y m n}
\end{array}\right]
\end{gathered}
$$

$K_{x m n}$ and $K_{y m n}$ are constants of normalization and defined as:

$$
K_{x m n}=\frac{m}{a} k_{m n} \quad \text { and } \quad K_{y m n}=\frac{n}{b} k_{m n}
$$

Where:

$$
k_{m n}=\frac{1}{\sqrt{\left(\frac{m}{a}\right)^{2}+\left(\frac{n}{b}\right)^{2}}}
$$

\subsection{THE DIFFRACTION OPERATOR $₫$ IN SPATIAL DOMAIN}

The global operator of diffraction near the whole interface is given by,

$\S_{\mathrm{int}}=\left[\begin{array}{cc}\frac{1-N^{2}}{1+N^{2}} H_{I}-H_{M}+\chi H_{S} & \frac{2 N}{1+N^{2}} H_{I}+\kappa H_{S} \\ \frac{2 N}{1+N^{2}} H_{I}+\xi H_{S} & \frac{1-N^{2}}{1+N^{2}} H_{I}-H_{M}+\zeta H_{S}\end{array}\right]$

where:

$$
\begin{gathered}
N=\sqrt{\frac{Z_{01}}{Z_{02}}} \text { and, } \\
H_{d}=\left\{\begin{array}{ll}
1 & \text { on the considered domain } \\
0 & \text { elsewhere }
\end{array} .\right.
\end{gathered}
$$

$d=I, M, S$ corresponds respectively to dielectric domains (insulating), metal and source and $\chi, \kappa, \xi, \zeta$ depend on the type of used source [12].

\subsection{THE REFLECTION OPERATOR $£$ IN MODAL DOMAIN}

The operator of modal reflection $\mathbb{E}$ insures the link between the waves diffracted at the interface $\vec{B}_{i}$ and the waves reflected by the superior and lower $\operatorname{hood} \vec{A}_{i}$.

It is expressed in the modal domain by the following equation [9],

$$
\mathrm{f}_{i}=\sum_{m, n, \alpha}\left|f_{m n}^{\alpha}\right\rangle \Gamma_{m n}^{\alpha, i}\left|f_{m n}^{\alpha}\right\rangle
$$

Where,

$$
\Gamma_{m n}^{\alpha, i}=\frac{1-Z_{0 i} Y_{m n}^{\alpha, i}}{1+Z_{0 i} Y_{m n}^{\alpha, i}}
$$


$f_{m n}^{\alpha}$ the mode functions of periodical wall near the discontinuity surface $(\Omega)$, $\alpha$ the mode index (TE, TM) and $Y_{m n}^{\alpha, i}$ is the mode of admittance brought back.

Where no closing ends exist, $Y_{m n}^{\alpha, i}$ can be calculated by :

$$
\begin{aligned}
& {\underset{i m n}{E^{T M}}}^{T M}=\frac{j \omega \varepsilon_{O} \varepsilon_{r i}}{\gamma_{m n}^{(i)}} \\
& \mathbb{i m n}_{i m}^{\mathbb{E}_{m n}^{T M}}=\frac{\gamma_{m n}^{(i)}}{j \omega \mu_{O}}
\end{aligned}
$$

$\gamma_{m n}^{(i)}$ being the propagation constant of the medium $i$ and it is given by

$$
\gamma_{m n}^{(i)}=\sqrt{\left(\frac{2 \pi m}{a}\right)^{2}+\left(\frac{2 \pi m}{a}\right)^{2}-k_{O}^{2} \varepsilon_{r i}}
$$

In equation (16), $\varepsilon_{\mathrm{o}}, \varepsilon_{\mathrm{ri}}$ and $\mu_{\mathrm{o}}$ are respectively the permittivity of the vacuum, the relative permittivity of the medium $i$, and the permeability of the vacuum.

When the structure along the $\mathrm{z}$ axis is terminated in the metallic wall (short circuit), the admittance seen by each mode at the interface is given by

$$
Y_{m n}^{i \alpha} c c=\xi_{m n}^{i i \alpha} \operatorname{coth}\left(\gamma_{m n}^{(i)} h_{i}\right)
$$

Where $\mathrm{h}_{i}$ is the substrate thickness of the medium $\mathrm{i}$. If a termination was an open circuit (no metallic wall at the end of the medium $i$ ), the admittance seen by each mode at the interface is given by

$$
Y_{m n}^{i \alpha} o c=\bigoplus_{m n}^{i \alpha} \operatorname{coth}\left(\gamma_{m n}^{(i)} h_{i}\right)
$$

\subsection{IMPEDANCE SEEN BY THE SOURCE}

For each iteration the impedance $Z_{\text {in }}$ seen by the source (respectively the $Y_{\text {in }}$ admittance) is computed according to the electromagnetic magnitudes. This factor justify at the end the convergence ever of the problem. It is determined by following variational relation,

$$
Z_{\text {in }}=\frac{1}{Y_{\text {in }}}=-\frac{\left\langle E_{o} \mid E_{o}\right\rangle}{\left\langle E_{o} \mid J\right\rangle}
$$

To use the electric device (R.F very high frequency, low-frequency or other), it is necessary to present a steady state during its use. From the convergence study of impedance $Z_{i n}$ which is seen by the source of excitation, the point of electric stability could be determined. This point must be chosen so that the convergence is always assured, regardless of the frequency.

\section{EXAMPLES OF PLANAR DEVICES}

In order to show that the iterative method is working accurately, it is better to illustrate the theory exposed previously by some applications. Let's look to the behavior of an antenna patch in microstrip and coplanar technologies, then to the application of directive quarter wavelength coupler [13], [14].

\subsection{PATCH ANTENNA WITH NOTCHES IN MICROSTRIP TECHNOLOGY}

\section{Structure description}

In Figure 3 a patch working around $1.32 \mathrm{GHz}$ is presented. It is composed with a line penetrating in the patch and with notches. The antenna which product a satisfactory adaptation presents at its extremity an input impedance of $50 \Omega$ and has the following dimensions,

$a=b=152 \mathrm{~mm}, w=4.75 \mathrm{~mm}, S=4.75 \mathrm{~mm}$

$L_{\text {fed }}=75.9 \mathrm{~mm}, L=5.9 \mathrm{~mm}, W=90.25 \mathrm{~mm}, Y_{0}=19 \mathrm{~mm}$

$h$ (thickness of substrat $)=0.5 \mathrm{~mm}, \varepsilon_{r}=2.3$

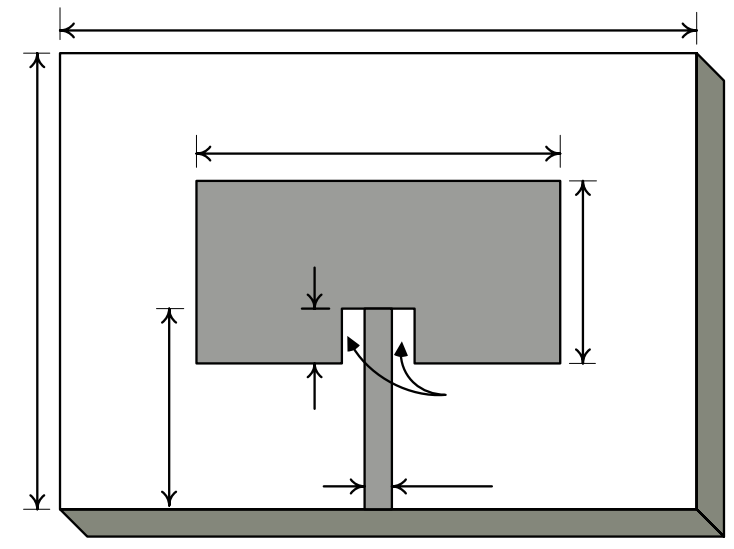

Fig. 3 - Patch antenna with notches

\section{Study of the convergence}

Observing the figure 4 , we set up the minimum number of iterations required to establish the final result. In our case the convergence is reached after 250 iterations at $1.32 \mathrm{Ghz}$. Moreover, it appears that the real part of the admittance of the patch seen by the source, converges to the value 0 which is relevant of a good convergence.

\section{Study of the S parameters}

In order to visualize the profile of the parameters $\mathrm{S}$ of the patch with respect to the frequency, the 
program has been iterated 300 times, following all the constraints of simulation. The obtained results are represented by the following graph:

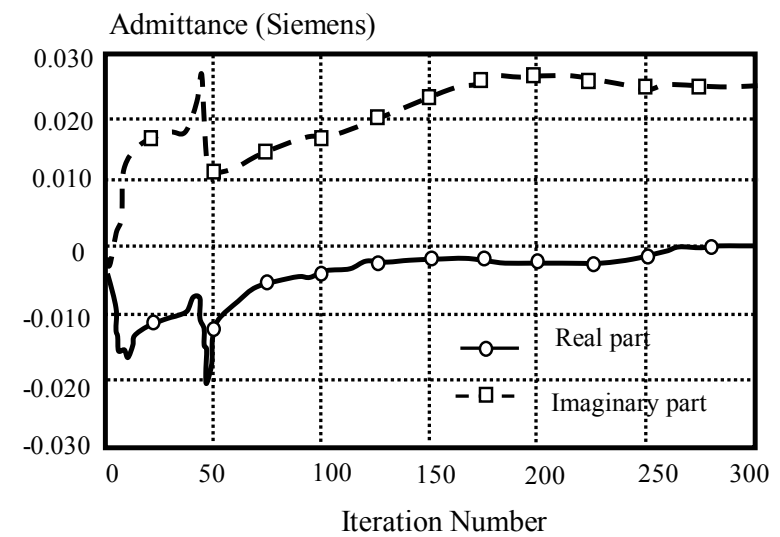

Fig. 4 - Convergence curves

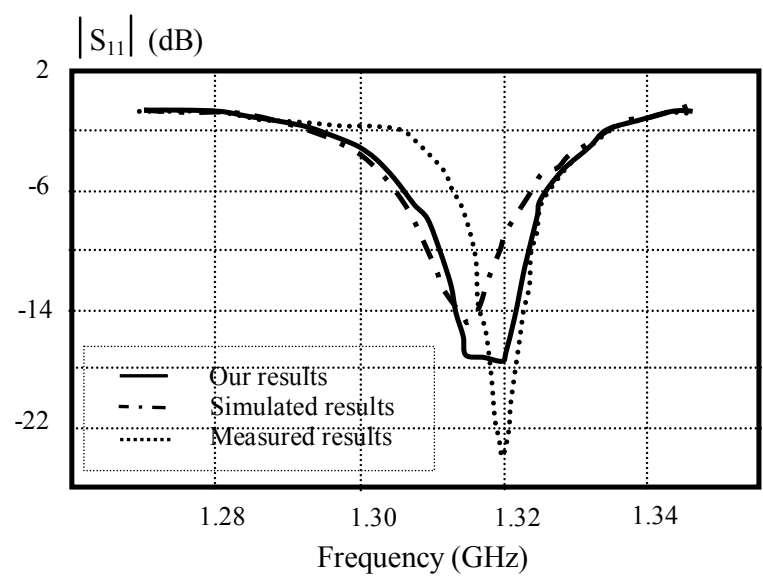

Fig. 5 - Module of S11 parameters versus frequency for a patch antenna with notches

The objective of the method is to obtain the resonant frequency equal to $1.32 \mathrm{GHz}$. We notice on our profile that the results ( Fig.5.) given by our method are close to this frequency (around 1.318), or even better than the result obtained from simulation [15]. Consequently, our method gives more reliable and precise results.

\subsection{PATCH ANTENNA IN COPLANAR TECHNOLOGY}

In this part, we present the study another structure given in figure 6, representing a patch antenna designed coplanar technology [15-19].

\section{Structure description}

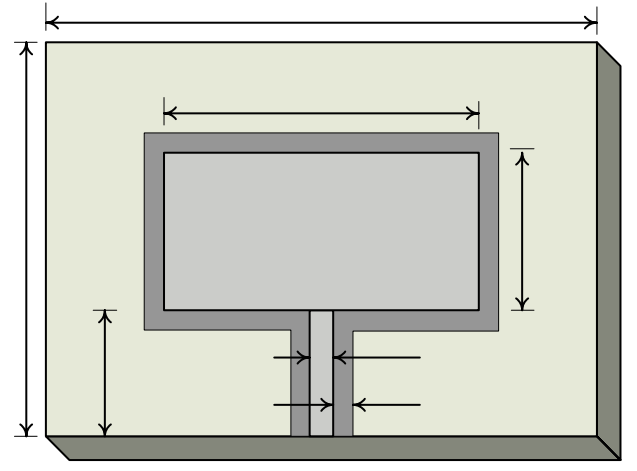

Fig. 6 - Coplanar patch antenna

The dimensions and parameters of the antennas are defined as follows [17],

$a=b=64 \mathrm{~mm}, w=1.6 \mathrm{~mm}, S=1.0 \mathrm{~mm}, \mathrm{~s}=1.0 \mathrm{~mm}$

$L_{\text {fed }}=171 \mathrm{~mm}, L=9.55 \mathrm{~mm}, W=31 \mathrm{~mm}, \varepsilon_{r}=2.17$

$h($ thickness of substrat $)=0.508 \mathrm{~mm}, Z_{c}=50 \Omega, f=10 \mathrm{Ghz}$

\section{Study of the S parameters}

The frequency dependency results are represented by Figure.7. It has been noticed through the obtained results that the resonant frequency of 10 $\mathrm{GHz}$ is well reached (our results). These results are in agreement with those obtained from experimental and simulated measures given in references [16].

We remark through the plots of the $\mathrm{S}$ parameters shown in Figures 5 and 7 that the results are very similar. The reason is that our method and the moments method use the same type of source, i.e. planar (horizontal) sources and metallic walls.

\subsection{QUARTER WAVELENGTH DIRECTIVE COUPLER}

In this part, the approach of the study for another structure shown in Figure 8 representing a directive quarter of wave coupler $[15,16]$. The dimensions of the coupler are as follows,

$a=b=25984 \mathrm{~mm},(m \times n)=(64 \times 64)$ pixels

$L_{1}=24 . \Delta x, w_{1}=6 . \Delta y, \varepsilon_{r 1}=1, h_{1}=0, L_{2}=18 . \Delta x$

$w_{2}=10 . \Delta y, \varepsilon_{r 2}=2.2, h_{2}=0.794 m m, w_{3}=6 . \Delta x$

\section{Study of the coupler}

The $\mathrm{S}$ parameters of directive coupler are examined as function of frequency with our program set for 300 iterations. According to these studies, the obtained results are shown in the figures 9, 10 and 11.

As shown in Figures 9, the $S_{11}$ coefficient is very weak at resonant frequency of $6.25 \mathrm{GHz}$, the coupler is adapted to this frequency. The $\mathrm{S}_{21}$ coefficient (Figure 10) is nearly zero at this resonant frequency, which mean that the line 2 is well isolated. The 
$\mathrm{S}_{13}$ and $\mathrm{S}_{14}$ coefficients (Figure 11) have the same magnitude at this resonant frequency. The power injected in the line (1) is devised in two identical powers toward the direct line 3 and the coupled line 4.

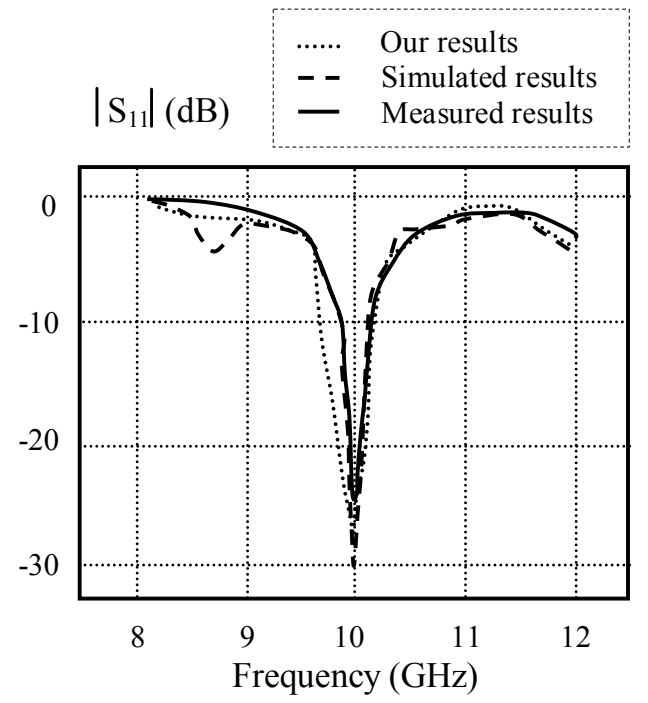

Fig.7 - Module of S11 parameters versus frequency for coplanar patch antenna

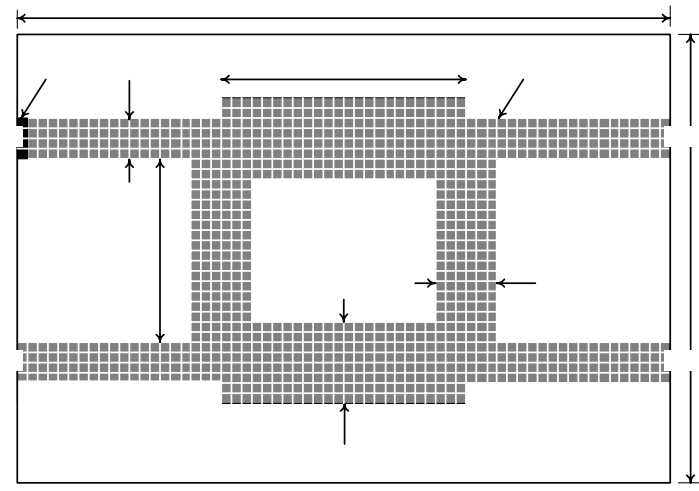

Fig. 8 - Quarter wavelength directive coupler

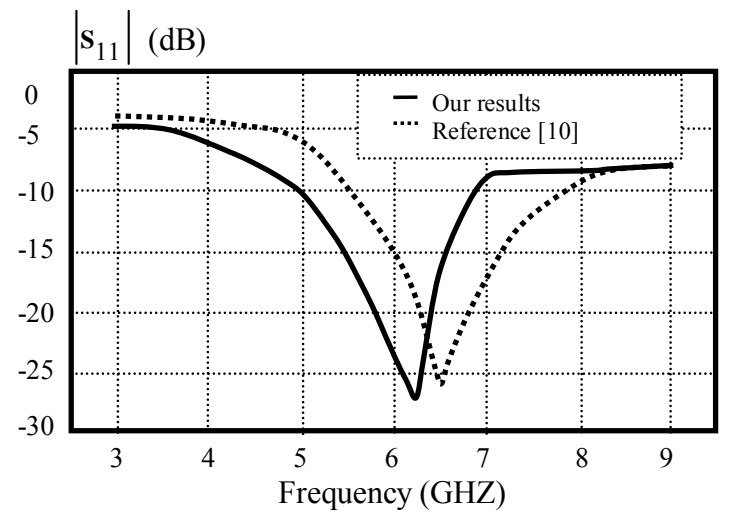

Fig. 9 - Module of S11 parameter versus frequency [10]

We remark through the plots of the S parameters of Figures 9 and 10, a slight difference in the resonant frequency. The reason for that lies in the different nature of the type of source as well as the walls of the box. Again, we point to the fact that the FDTD method uses vertical sources and absorbent walls.

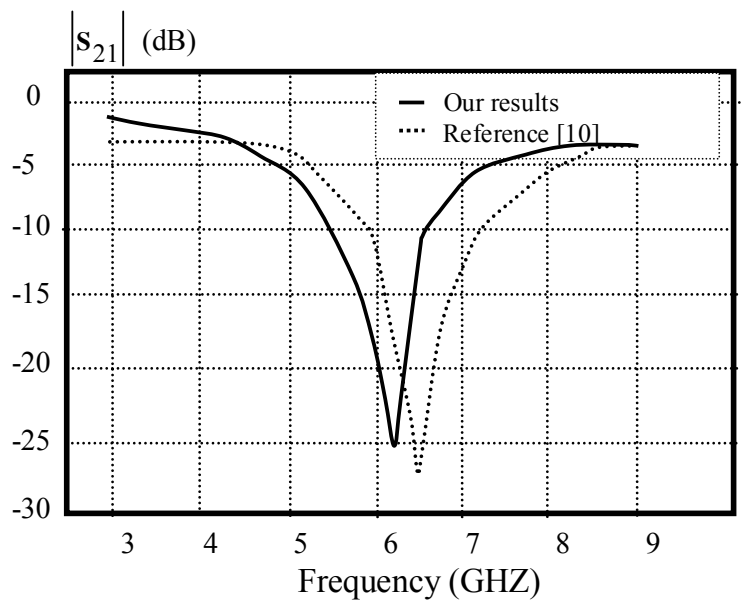

Fig. 10 - Module of S21 parameter versus frequency

$$
\begin{aligned}
& -\left|\mathbf{S}_{14}\right| \text { Our results, } \ldots-\left|\mathbf{S}_{14}\right| \text { Reference [10] } \\
& =--\left|\mathbf{S}_{13}\right| \text { Our results, } \ldots . .\left|\mathbf{S}_{13}\right| \text { Reference [10] }
\end{aligned}
$$

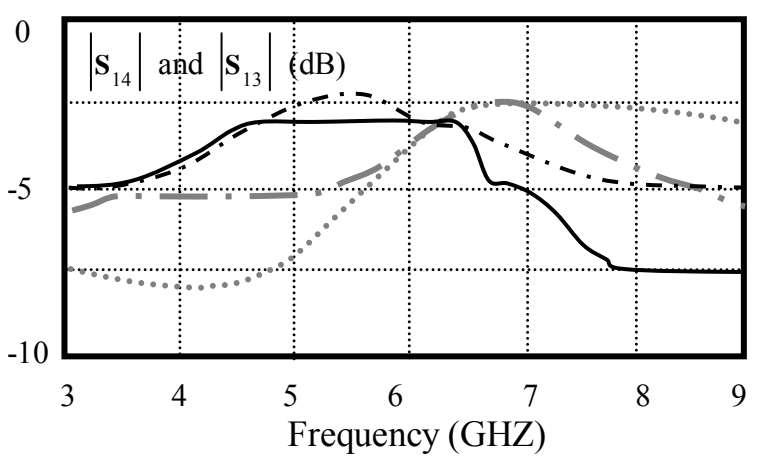

Fig. 11 - S13 and S14 parameters versus frequency

\section{CONCLUSION}

In this paper the WCIP has been applied successfully to planar microwave circuits. The method has been extended to the modeling of path antenna. Its principles and properties have been described to show its flexibly against other techniques. The contribution of the FFT allows a fast processing and avoids the delicate choice of functions that is usually met in other integral methods. Hence, the WCIP seen to be very easy to implement, reliable in term of convergence and has the possibility to treat problems with large number of undefined parameters as the FFT is written without storing its matrix in memory. 
The WCIP were applied with success to model two patches in microstrip and coplanar technologies and to a directive quarter wavelength coupler. The WCIP results and those previously reported in the literature are in very good agreement. This method seems highly suited for use on planar structures; its extension to problems of any shape is under investigation. The perspective of the method is to be applied to metamaterial, integrated circuits on subtract and to circuits with large number of layers including a large number of parameters.

\section{NOMENCLATURE}

\begin{tabular}{|ll|}
\hline$\Omega_{i}$ & Interfacedi continuity \\
$n_{i}$ & Normal vector \\
$i$ & Indice of the medium \\
$J_{i}$ & Current density \\
$H_{i}$ & Magnetic field \\
$E_{i}$ & Electric field \\
$A_{i}$ & Reflected wave \\
$B_{i}$ & Incident wave \\
$Z_{0 i}$ & Impedance of wave \\
$\mathbb{E}$ & Operator of reflection \\
$\oiint_{1}$ & Operator of diffraction \\
$B_{0}$ & Excitation wave \\
$k_{m n x}$, & Constants of Propagation \\
$k_{m n y}$ & \\
$f_{m n}^{\alpha}$ & Basis mode function \\
$Y_{m n}^{\alpha, i}$ & Mode admittance \\
$h_{i}$ & Height \\
$Z_{i n}$ & Impedance seen by source \\
\hline
\end{tabular}

\section{ACKNOWLEDGMENTS}

This work was supported by the Ministère de l'Enseignement Supérieure et de la Recherche Scientifique (MESRS) under the supervising of CNEPRU project (www.cnepru-mesrs.dz).

The two first authors would like to thank the staff of GRE (Groupe de Recherche en Electromagnétisme) of the ENSEEIHT de Toulouse (INPT, France) for theirs helps during the period of training.

All my gratitude to Professor Mokhtar Attari, Head of the laboratory of instrumentation (LINS) for his precious help regarding to the manuscript outline.

\section{REFERENCES}

[1] M. Azizi, M. Boussouis, H. Aubert, and H. Budrand, "A Three-Dimensional Analysis of
Planar Discontinuities by an Iterative Method" Microwave and Optical Technology Letters, vol. 13, pp. 372-376, Dec. 1996.

[2] Christopoulos, C.: 'The transmission line modeling method: TLM', IEEE Press, Piscataway, NJ, 1995.

[3] Russer, P., and Bader, B.: 'The alternating transmission line matrix (ATLM) scheme'. IEEE MTT-S Proc. Int. Microw. Symp., Orlando, FL, 1995 May 16-20, pp. 19-22

[4] Taflove, A., and Hagness, S.: Computational electrodynamics: the finite-difference timedomain method, Artech House, Norwood, 2000.

[5] Baudrand, H., and Bajon, D.: 'Equivalent circuit representation for integral formulation of electromagnetic problems', Int. J. Numer., Model. Electron. Netw. Devices Fields, January 2002, 15, pp. 23-57

[6] Baudrand, H., and N'Gongo, R.S.: 'Application of wave concept iterative procedure', Recent Research Developments in Microwave Theory and Techniques, Transworld Research Network, Kerala, India, 1999), Vol. 1, pp. 187-197

[7] Akatimagool, S., Bajon, D., and Baudrand, H.: 'Analysis of multilayer integrated inductors with wave concept iterative procedure (WCIP)'. IEEE MTT-S Proc. Int. Microw. Symp. Dig., May 2001, Vol. 3, pp. 1941-1944

[8] Wane, S., Bajon, D., and Baudrand, H.: 'Fullwave analysis of stacked structures including inhomogeneous layers. Hybrid Integral-Differential Approach in Terms ofWaves'. Proc. New Trends and Concepts in Microwave Theory and Techniques Conf., February 2003, pp. 131-166

[9] R.S. N'gongo and H. Baudrand, "Application of wave concept iterative procedure in planar circuit," Recent Res. Devel. Microwave theory \& Tech., Vol.1, pp. 187-197, 1999.

[10] M. David , M. A. Sheen, D. Mohamed and J. $\mathrm{Au}$ Kong, "Application of the threedimensional finite-difference time-domain method to the analysis of planar microstrip circuit," IEEE Trans on microwave theory and techniques, vol 38, $\mathrm{n}^{\circ}$ 7, pp.849-856, July. 1990.

[11] R. Garcia, Contribution à l'Etude des Circuits Planaires par une Méthode Itérative Basée sur le Concept d'Ondes, Thèse de doctorat, ENSEEIHT Toulouse, 2001.

[12] Arun K. Bhattacharyya, "Long rectangular patch antenna with a single feed," IEEE Transaction on antennas propagation, Vol.38, No 7, July 1990. 
[13] Davic M. Pozar, "Microstrip Antennas," IEEE Transaction on Antennas and Propagation, VOL. 80, Nº1, January 1992.

[14] Keith R. Carver and James W. Mink, "Microstrip Antennas Technology," IEEE Transaction on Antennas and Propagation, Vol.29, No. 1, January 1981.

[15] K. Li, C. H. Cheng, T. Matsui, and M. Izutsu, "Simulation and experimental study on coplanar patch and array antennas," IEEE Microwave Confernce, 2000 Asia-Pacific, 2000.

[16] F. Surre, L. Cohen, H. Baudrand, P. Gamand, "New Approaches for Multi-Scale circuit Analysis", EUMC 2001, Londre (G.R), September 2001

[17] H. Baudrand, Introduction au calcul des circuits microsondes, CEPADUES Ed., ENSEEIHT, Toulouse, 1994.

[18] Ludovic Cohen, Contribution à l'Etude et à la Modélisation de Circuits Coplanaires Multicouches par une Méthode Itérative, Doctorat thesis, INPT, France, 2002.

[19] W.J. Tseng et S.J. Chung , "Analysis and Application of a Two-port Aperture-coupled Microstrip Antenna," IEEE Trans Microwave Theory Tech, Vol 46, pp.530-535, Mai 1998.

[20] Tellache, B.Haraoubia, H.Baudrand, "Modélisation des circuits planaires par la méthode itérative," $19^{\text {ème }}$ Conférence Internationale Optique Hertzienne et Diélectrique, OHD 2007, $05-08$ Septembre 2007, Valence, France.

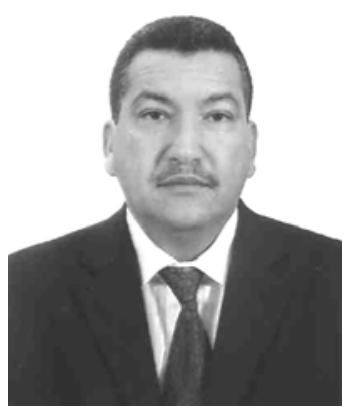

Mohamed Tellache was born in Draa El Mizane, Algeria in 1960. He received the Engineer degree in Electrical Engineering from the Ecole Nationale Polytechnique of Algiers in 1985. He received his Master degree in 1988 in electronic communication from Ottawa University, Ottawa, Canada. During 1995/1997, Mr. Tellache spent eighteen months at the laboratory of electromagnetics-Toulouse, France working principally on microwaves technology and modeling. Presently he is Assistant Professor of Electrical Engineering and Adjoin Dean at the faculty of Electronics and Computers.

His present research interests focus on microwaves circuits, modeling and applied mathematics. Mr. Tellache is author and co- author of more than 15 communications in the field.

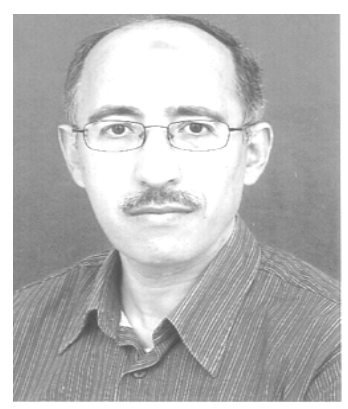

Youssef Lamhene received the Bachelor and Master degree in Electrical Engineering (Licence et Maîtrise EEA), in 1978 and 1980 respectively from Lille University, France. Also, he received the $D E A$ degree and the Doctorate degree (3ème Cycle) in Biotechnology in 1982 and 1984 respectively from the same university. Since 1986 he join the institute of electronics at Houari Boumediene University, Algiers where he teaches courses in applied electronic, microwave and antenna theory. His research interests are in the area of electromagnetic simulations of RF and microwave passive components. field.

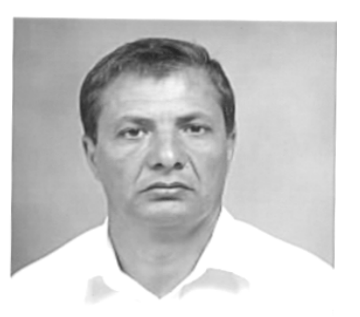

Brahim Haraoubia was born in Taoura, Algeria in 1957. He received the Engineer degree in Electrical Engineering from the Ecole Nationale Polytechnique of Algiers in 1981. He received his Master and PhD degrees in 1982 and 1988 both in electronic communication from Université de Rennes, France. Presently he is full Professor of Electrical Engineering at the faculty of Electronics and Computers.

His present research interests focus on microwaves circuits and modeling VHF/UHF circuits. Professor Haraoubia is author and coauthor of more than 30 publications and communications in the field and has deposit five patents in his field.

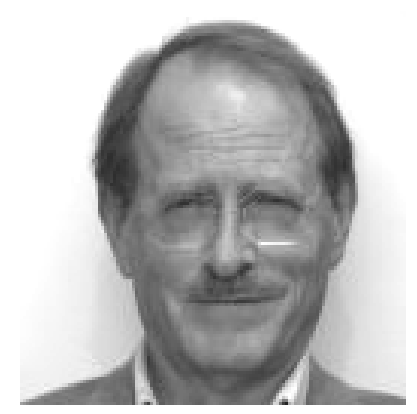

Henri Baudrand is Emeritus Professor at ENSEEIHT, Toulouse, France. He specializes in modelling passive and active circuits and antennas. He is the author and coauthor of three books at Cepadues edition. He has co-authored over 120 publications in journals, 4 chapters in 
scientific books, and 250 contributions to international conferences. $\mathrm{He}$ is a fellow member of IEEE society, a fellow member of "Electromagnetism Academy" and a senior member of IEE society. He was the president of URSI, France commission B for six years (1993 to 1999) and the president of the IEEE-MTT-ED French chapter. He was awarded "officier des palmes académiques" by French ministry of education and Director honoris causa of Lasi University in Romania. 